\title{
Near-infrared interferometric observation of the Herbig Ae star HD 144432 with VLTI/AMBER ${ }^{\star}$
}

\author{
L. Chen ${ }^{1}$, A. Kreplin ${ }^{1, \star \star}$, Y. Wang ${ }^{1}$, G. Weigelt ${ }^{1}$, K.-H. Hofmann ${ }^{1}$, S. Kraus ${ }^{2}$,

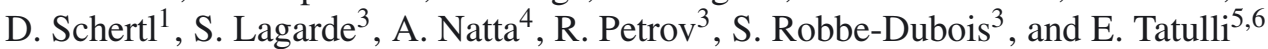 \\ ${ }^{1}$ Max-Planck-Institut für Radioastronomie, Auf dem Hügel 69, 53121 Bonn, Germany \\ e-mail: lchen@mpifr-bonn.mpg. de \\ 2 Department of Astronomy, University of Michigan, 500 Church St., Ann Arbor, MI 48109-1090, USA \\ ${ }^{3}$ Laboratoire Lagrange, UMR7293, Université de Nice Sophia-Antipolis, CNRS, Observatoire de la Côte d'Azur, 06300 Nice, France \\ 4 INAF - Osservatorio Astrofisico di Arcetri, Largo E. Fermi 5, 50125 Firenze, Italy \\ 5 UJF-Grenoble 1/CNRS-INSU, Institut de Planétologie et d'Astrophysique de Grenoble (IPAG) UMR 5274, 38041 Grenoble, France \\ ${ }^{6}$ Inter-University Centre for Astronomy and Astrophysics (IUCAA), Post Bag 4, Ganeshkhind, 411007 Pune, India
}

Received 13 January 2012 / Accepted 15 March 2012

\section{ABSTRACT}

\begin{abstract}
Aims. We study the sub-AU-scale circumstellar environment of the Herbig Ae star HD 144432 with near-infrared VLTI/AMBER observations to investigate the structure of its inner dust disk.

Methods. The interferometric observations were carried out with the AMBER instrument in the $H$ and $K$ band. We interpret the measured $H$ - and $K$-band visibilities, the near- and mid-infrared visibilities from the literature, and the spectral energy distribution (SED) of HD 144432 by using geometric ring models and ring-shaped temperature-gradient disk models with power-law temperature distributions.

Results. We derive a $K$-band ring-fit radius of $0.17 \pm 0.01 \mathrm{AU}$ and an $H$-band radius of $0.18 \pm 0.01 \mathrm{AU}$ (for a distance of $145 \mathrm{pc}$ ). This measured $K$-band radius of $\sim 0.17$ AU lies in the range between the dust sublimation radius of $\sim 0.13$ AU (predicted for a dust sublimation temperature of $1500 \mathrm{~K}$ and gray dust) and the prediction of models including backwarming $(\sim 0.27 \mathrm{AU})$. We find that an additional extended halo component is required in both the geometric and temperature-gradient modeling. In the best-fit temperaturegradient model, the disk consists of two components. The inner part of the disk is a thin ring with an inner radius of $\sim 0.21 \mathrm{AU}$, a temperature of $\sim 1600 \mathrm{~K}$, and a ring thickness $\sim 0.02 \mathrm{AU}$. The outer part extends from $\sim 1$ AU to $\sim 10$ AU with an inner temperature of $\sim 400 \mathrm{~K}$. We find that the disk is nearly face-on with an inclination angle of $<28^{\circ}$.

Conclusions. Our temperature-gradient modeling suggests that the near-infrared excess is dominated by emission from a narrow, bright rim located at the dust sublimation radius, while an extended halo component contributes $\sim 6 \%$ to the total flux at $2 \mu \mathrm{m}$. The mid-infrared model emission has a two-component structure with $\sim 20 \%$ of the flux originating from the inner ring and the rest from the outer parts. This two-component structure is indicative of a disk gap, which is possibly caused by the shadow of a puffed-up inner rim.
\end{abstract}

Key words. accretion, accretion disks - techniques: interferometric - protoplanetary disks - circumstellar matter stars: pre-main sequence - stars: individual: HD 144432

\section{Introduction}

HD 144432 (He3-1141) is an isolated Herbig Ae (HAE) star with spectral type A9/F0 (Thé et al. 1994; Sylvester et al. 1996) located at $\sim 145$ pc (Pérez et al. 2004; see also the discussion in Sect. 4). Since it is not closely associated with molecular cloud material (Malfait et al. 1998), its IR excess can be attributed to circumstellar (CS) material. By analyzing its spectral features, and considering its lack of photometric variability, Meeus et al. (1998) concluded that the central star of HD 144432 is directly seen, without CS material in the line of sight. Thus, they suggested that the CS material is confined to a face-on disk, instead of having a spherical geometry. HD 144432 belongs to the group II objects in the classification scheme by

* Based on observations made with ESO telescopes at Paranal Observatory under program ID 083.D-0224(C) and 085.C-0126(A).

$\star \star$ Member of the International Max Planck Research School (IMPRS) for Astronomy and Astrophysics at the Universities of Bonn and Cologne.
Meeus et al. (2001), i.e., it has a flat IR spectrum and a weaker mid-infrared (MIR) excess than the group I objects. A plausible explanation of a group II SED is that the dust disk is shadowed by its own puffed-up inner rim (Natta et al. 2001; Dullemond et al. 2001; Dullemond 2002; Dominik et al. 2003; Dullemond \& Dominik 2004). The polycyclic-aromatic-hydrocarbon emission features in HD 144432 are weak (Acke \& van den Ancker 2004; Keller et al. 2008), which supports the self-shadowed-disk interpretation.

Previous interferometric observations of HD 144432 in the $H$ and $K$ bands using IOTA and the Keck Interferometer (KI) were reported by Monnier et al. (2005, hereafter M05, 2006, hereafter M06), and Eisner et al. (2009). Spectrally dispersed interferometric $K$-band observations by Eisner et al. (2009) show that the size of the emission region increases with wavelength. Their modeling of the data suggests a dust disk with an inner radius of $0.25-0.3 \mathrm{AU}$ (at $145 \mathrm{pc}$ ) and an inner temperature of $1000-1200 \mathrm{~K}$, a gas disk between the star and the dust, and a $\mathrm{Br} \gamma$ line originating from a more compact region. In the MIR, 
Leinert et al. (2004) measured the half-light radius of the disk at $12.5 \mu \mathrm{m}$ to 14 mas ( 2 $\mathrm{AU}$ at $145 \mathrm{pc}$ ) using VLTI/MIDI. With Keck segment-tilting, Monnier et al. (2009) measured the Gaussian FWHM of HD 144432 to $39 \pm 5$ mas $(5.6 \pm 0.7 \mathrm{AU}$ at $145 \mathrm{pc})$ at $10.7 \mu \mathrm{m}$.

Pérez et al. (2004) found that the object is a binary, with a K-type T Tauri star companion at a separation of 1.4", and estimated the age of the two stars to 1-3 Myr. Carmona et al. (2007) confirmed that the primary and the companion are physically associated, but estimated the age to $8 \mathrm{Myr}$.

In this paper, we present $H$ - and $K$-band VLTI/AMBER observations of HD 144432. In Sect. 2, we summarize the observations and the data reduction. The modeling is presented in Sect. 3. We discuss the modeling results in Sect. 4 and present the summary and conclusion in Sect. 5.

\section{Observation and data reduction}

AMBER is the near-infrared (NIR) beam combiner instrument of the Very Large Telescope Interferometer (VLTI) and records spectrally dispersed three-beam interferograms, capable of measuring both visibilities and closure phases (CPs) (Petrov et al. 2007). HD 144432 was observed in the low spectral resolution mode $(R=35)$ in the $H$ and $K$ bands on 2009 Apr. 18 and 2010 Apr. 18 with VLTI/AMBER using the linear baseline configuration $\mathrm{E} 0-\mathrm{G} 0-\mathrm{H} 0$ and the triangle configuration $\mathrm{D} 0-\mathrm{H} 0-\mathrm{G} 1$, respectively (see Table 1). The visibilities and CPs are derived using the pixel-to-visibility-matrix (P2VM) algorithm of the data reduction package amdlib $3.0^{1}$. In Fig. 1, we show the $H$ - and $K$-band visibilities as a function of projected baseline length. The $1.4^{\prime \prime}$ binary companion has no influence on the visibility measurements of the primary star, since the field-of-view of the AMBER is only $0.25^{\prime \prime}$. The extracted CPs are shown in Fig. 2 and have wavelength-averaged values of $1.5 \pm 1.7^{\circ}$ (2009), $0.3 \pm 5.0^{\circ}$ (2010a), and $-3.1 \pm 5.9^{\circ}$ (2010b). In Fig. 3a (upper three rows), we present the derived wavelength-dependent AMBER visibilities of HD 144432 in the $H$ and $K$ bands. For data processing, we selected $30 \%$ of the frames with the highest fringe signal-to-noise ratio (Tatulli et al. 2007) of each target and calibrator data set. From the 2009 and 2010a data (see Table 1), we extracted both $H$ - and $K$-band visibilities and CPs. From the 2010 b data, only $K$-band visibilities and CPs were extracted owing to the low fringe signal-to-noise ratio of the $H$-band data. We used the method of OPD histogram equalization (Kreplin et al. 2012) to reduce the influence of the atmospheric optical path differences (OPDs) on the calibrated visibilities. In the calibration process, we used in both nights the calibrator star HD 142669 with an uniform disk diameter of $d_{\mathrm{UD}}=0.27 \pm 0.05 \mathrm{mas}^{2}$.

\section{Modeling}

We attempt to build models that can reproduce both the data from our new observations and data sets available in literature, including the $K$-band observation with KI (M05, Eisner et al. 2009), the $H$-band observation with IOTA (M06), the MIR interferometry with VLTI/MIDI (Leinert et al. 2004), as well as the SED (Leinert et al. 2004). In the modeling process, we assume a distance of 145 pc (Pérez et al. 2004).

\footnotetext{
1 Available at: http://www.jmmc.fr/data_processing_amber. htm

2 Taken from the Catalogue of Stellar Diameters (CADARS) (Pasinetti Fracassini et al. 2001).
}
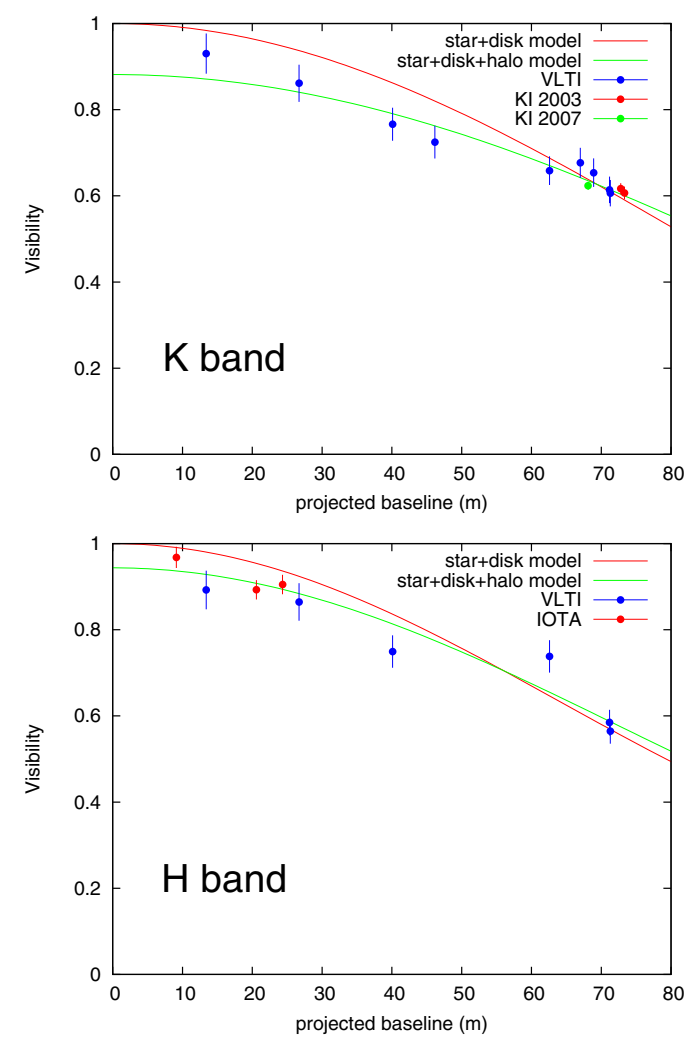

Fig. 1. Band-averaged visibilities as a function of baseline length. The dots with errorbars are the observations (blue dots: our VLTI data; red and green dots: IOTA and KI data taken from Monnier et al. 2005, 2006; and Eisner et al. 2009). The lines are best-fit geometric models (inclination $0^{\circ}$, red: star-disk model; green: star-disk-halo model). Top panel: $K$ band. Bottom panel: $H$ band. Model parameters are listed in Table 3.

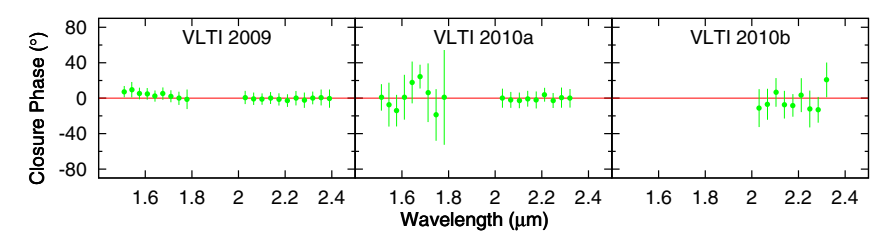

Fig. 2. Observed HD 144432 closure phases as a function of wavelength.

\subsection{Geometric modeling}

First, simple geometric models are employed to characterize the size of the disk. In order to be consistent with M05's work, we used the uniform-brightness ring model, which has a ring thickness of $20 \%$ of the inner radius $R_{\text {ring }}$. By fitting a Kurucz model (Kurucz 1992) to the stellar component of the dereddened SED (taken from Leinert et al. 2004) and measuring the flux excess in the $H$ and $K$ bands, we obtained the ring (disk) flux fractions $f_{\text {ring }}=0.54$ for the $H$ band and $f_{\text {ring }}=0.73$ for the $K$ band, respectively.

The wavelength-averaged $H$ - and $K$-band visibilities were fitted with a ring model including the stellar contribution. The total visibility of the star-ring model can be described by

$V_{\text {total }}=f_{\text {ring }} V_{\text {ring }}+f_{*} V_{*}$,

where $V_{\text {total }}$ is the measured visibility, $f_{*}=1-f_{\text {ring }}$ is the flux contribution from the central star, and $V_{*}$ is the stellar visibility. As the central star is unresolved $\left(R_{*}<0.1 \mathrm{mas}\right)$, we set $V_{*}=1$. The visibility $V_{\text {ring }}$ of an uniform ring is calculated following 
Table 1. Observation log of our VLTI/AMBER observation of HD 144432.

\begin{tabular}{lccccccc}
\hline \hline Data set & Night & $\begin{array}{c}t_{\text {obs }} \\
(\mathrm{UTC})\end{array}$ & Telescope configuration & $\begin{array}{c}B_{\mathrm{p}} \\
(\mathrm{m})\end{array}$ & $\begin{array}{c}\text { PA } \\
\left({ }^{\circ}\right)\end{array}$ & $\begin{array}{c}\text { Seeing } \\
\left({ }^{\prime \prime}\right)\end{array}$ & $\begin{array}{c}\text { DIT }^{a} \\
(\mathrm{~ms})\end{array}$ \\
\hline 2009 & $2009-04-18$ & $04: 02: 36$ & E0-G0-H0 & $13.4 / 26.7 / 40.1$ & 41.7 & 0.72 & 200 \\
$2010 \mathrm{a}$ & $2010-04-18$ & $07: 57: 31$ & D0-H0-G1 & $62.5 / 71.2 / 71.3$ & $76.9 / 140.6 / 12.8$ & 0.75 & 200 \\
2010b & $2010-04-18$ & $10: 20: 02$ & D0-H0-G1 & $46.2 / 67.0 / 68.9$ & $92.4 / 164.8 / 24.5$ & 0.59 & 200 \\
\hline
\end{tabular}

Notes. ${ }^{(a)}$ Detector integration time.

Table 2. Best-fit parameters of our geometric modeling.

\begin{tabular}{|c|c|c|c|c|c|c|c|c|}
\hline Model & Band & $R_{\text {ring }}(\mathrm{AU})$ & $f_{\text {ring }}$ & $f_{\text {star }}$ & $f_{\text {halo }}$ & $i\left(^{\circ}\right)$ & $\theta_{\mathrm{D}}\left(^{\circ}\right)$ & $\chi_{\mathrm{red}}^{2}$ \\
\hline \multirow{2}{*}{ star-ring } & $\bar{K}$ & $0.210 \pm 0.008$ & 0.73 & 0.27 & 0 & & & 2.3 \\
\hline & $H$ & $0.204 \pm 0.011$ & 0.54 & 0.46 & 0 & & & 3.4 \\
\hline \multirow[t]{2}{*}{ star-ring-halo } & $\bar{K}$ & $0.169 \pm 0.009$ & 0.73 & $0.15 \pm 0.02$ & $0.12 \pm 0.02$ & & & 0.8 \\
\hline & $H$ & $0.180 \pm 0.010$ & 0.54 & $0.40 \pm 0.02$ & $0.06 \pm 0.02$ & & & 1.6 \\
\hline star-ring & $K$ & $0.223 \pm 0.015$ & 0.73 & 0.27 & 0 & $26 \pm 12$ & $-81 \pm 16$ & 2.5 \\
\hline (inclined) & $H$ & $0.218 \pm 0.018$ & 0.54 & 0.46 & 0 & $34 \pm 16$ & $17 \pm 25$ & 3.9 \\
\hline star-ring-halo & $K$ & $0.178 \pm 0.014$ & 0.73 & $0.16 \pm 0.03$ & $0.11 \pm 0.03$ & $24 \pm 10$ & $-86 \pm 16$ & 0.8 \\
\hline (inclined) & $H$ & $0.192 \pm 0.012$ & 0.54 & $0.40 \pm 0.02$ & $0.06 \pm 0.02$ & $37 \pm 11$ & $1 \pm 15$ & 1.5 \\
\hline
\end{tabular}

Notes. $R_{\text {ring }}$ is the inner radius of the ring-shaped disk models. $f_{\text {ring }}, f_{\text {star }}$, and $f_{\text {halo }}$ denote the flux contributions of the ring, the star, and the halo, respectively. $i$ is the inclination angle $\left(0^{\circ}\right.$ corresponds to a face-on ring). $\theta_{\mathrm{D}}$ is the position angle of the ring's major axis. $\chi_{\text {red }}^{2}$ is the reduced chi-square.

Table 3. Best-fit parameters of the temperature-gradient models.

\begin{tabular}{ccccccccccccc}
\hline \hline & $k_{\text {halo }}$ & $i\left(^{\circ}\right)$ & $\theta_{\mathrm{D}}\left({ }^{\circ}\right)$ & $T_{1, \text { in }}(\mathrm{K})$ & $q_{1}$ & $r_{1, \text { in }}(\mathrm{AU})$ & $\Delta r_{1}(\mathrm{AU})$ & $T_{2, \text { in }}(\mathrm{K})$ & $q_{2}$ & $r_{2, \text { in }}(\mathrm{AU})$ & $\Delta r_{2}(\mathrm{AU})$ & $\chi_{\text {red }}^{2}$ \\
\hline one-component & 0.306 & 60 & 33 & 800 & 0.63 & 0.392 & 5.0 & & & & \\
\hline two-component & $0.185_{-0.013}^{+0.013}$ & $10_{-10}^{+8 a}$ & $30_{-120}^{+60}$ & $1567_{-29}^{+55}$ & $0.5^{b}$ & $0.214_{-0.003}^{+0.005}$ & $0.019_{-0.002}^{+0.001}$ & $392_{-7}^{+6}$ & $0.85_{-0.04}^{0.01}$ & $0.93_{-0.03}^{+0.02}$ & $7.9_{-0.5}^{+0.5}$ & 1.15 \\
\hline
\end{tabular}

Notes. ${ }^{(a)}$ With $99.7 \%$ confident limit $i<28^{\circ} .{ }^{(b)}$ The power-law index $q_{1}$ was fixed to 0.5 (corresponding to a flared irradiated disk; Kenyon \& Hartmann 1987), since $q_{1}$ cannot be constrained because of the small radial thickness of the inner disk ring (see Appendix A.3).

Eq. (8) in Eisner et al. (2004). The best-fit geometric star-ring models are presented in Fig. 1 and Table 2 (for a distance of $145 \mathrm{pc}$, Pérez et al. 2004). We derived ring-fit radii of $R_{\text {ring }}=$ $0.21 \pm 0.01 \mathrm{AU}$ for the $K$ band and $R_{\text {ring }}=0.20 \pm 0.01 \mathrm{AU}$ for the $H$ band.

The high reduced chi-square errors $\chi_{\text {red }}^{2}$ of the visibility fits (see last column in Table 2) indicate that even the best-fit starring models cannot reproduce the observational data well. M06 reported that for some Herbig Ae stars an additional overresolved halo component (i.e., a halo that is too large to be constrained by the short baselines) is required to permit us to fit the visibilities with simple geometric models. Therefore, we introduce a halo component into our model. We assume that the halo structure scatters the light from the central star. While $f_{\text {ring }}$ remains the same parameter as above, $f_{*}$ is given by $f_{*}=$ $1-f_{\text {ring }}-f_{\text {halo }}$, where the halo flux ratio, $f_{\text {halo }}$, is an additional free parameter. The best-fit star-ring-halo model in the $K$ band shows that $12 \pm 2 \%$ of the flux is emitted by the halo, and that the ring has a ring-fit radius of $R_{\text {ring }}=0.17 \pm 0.01 \mathrm{AU}$. In the $H$ band, the halo contributes $6 \pm 2 \%$ to the total flux and the ring-fit radius is $0.18 \pm 0.01 \mathrm{AU}$. With an additional halo component, the values of the $\chi_{\text {red }}^{2}$ decrease by a factor of $\sim 2.5$ (see Table 2 ).

The measurements at different position angles allow us to investigate the inclination of the disk. Thus, we also employed inclined ring models with and without an extended halo. The fitting results are summarized in Table 2. The best-fit models suggest small inclination angles of $\sim 30^{\circ}$, but the $\chi_{\text {red }}^{2}$ values do not show a significant decrease.

Finally, to roughly characterize the $N$-band size, we fitted the band-averaged visibility of $0.19 \pm 0.1$ with a thin-ring model and derived a ring radius of $\sim 1.4 \mathrm{AU}$. We also studied a geometric two-ring model and derived a fit radius of $1.6 \mathrm{AU}$ for the outer ring, by assuming an estimated $N$-band flux contribution of $15 \%$ from the inner model ring and an inner-ring radius of $0.18 \mathrm{AU}$, derived in the above star-ring-halo model (see Table 2).

\subsection{Temperature-gradient modeling with a one-component disk}

Temperature-gradient models are employed to fit the NIR and MIR visibilities as well as the SED data simultaneously. We begin with a simple model including a star, an inclined onecomponent disk, and an extended overresolved halo. The star contributes to the flux $F_{*}(\lambda)=A_{*} I_{*}(\lambda)$, where $I_{*}(\lambda)$ is the intensity at the stellar surface and $A_{*}=\pi R_{*}^{2}$ is the angular area of the star. The halo is assumed to be very extended, emitting a spectrum that is similar to the stellar spectrum. Thus, its flux is $F_{\text {halo }}(\lambda)=k_{\text {halo }} F_{*}(\lambda)$. The disk is an optically thick ring with a power-law temperature distribution (Hillenbrand et al. 1992) $T=T_{\text {in }}\left(r / r_{\text {in }}\right)^{-q}, r_{\text {in }} \leq r \leq r_{\text {out }}$, with the inner and outer radius $r_{\text {in }}$ and $r_{\text {out }}$ of the disk, a disk temperature $T_{\text {in }}$ at $r_{\text {in }}$, and a power-law index $q$. Each part of the ring emits black body 

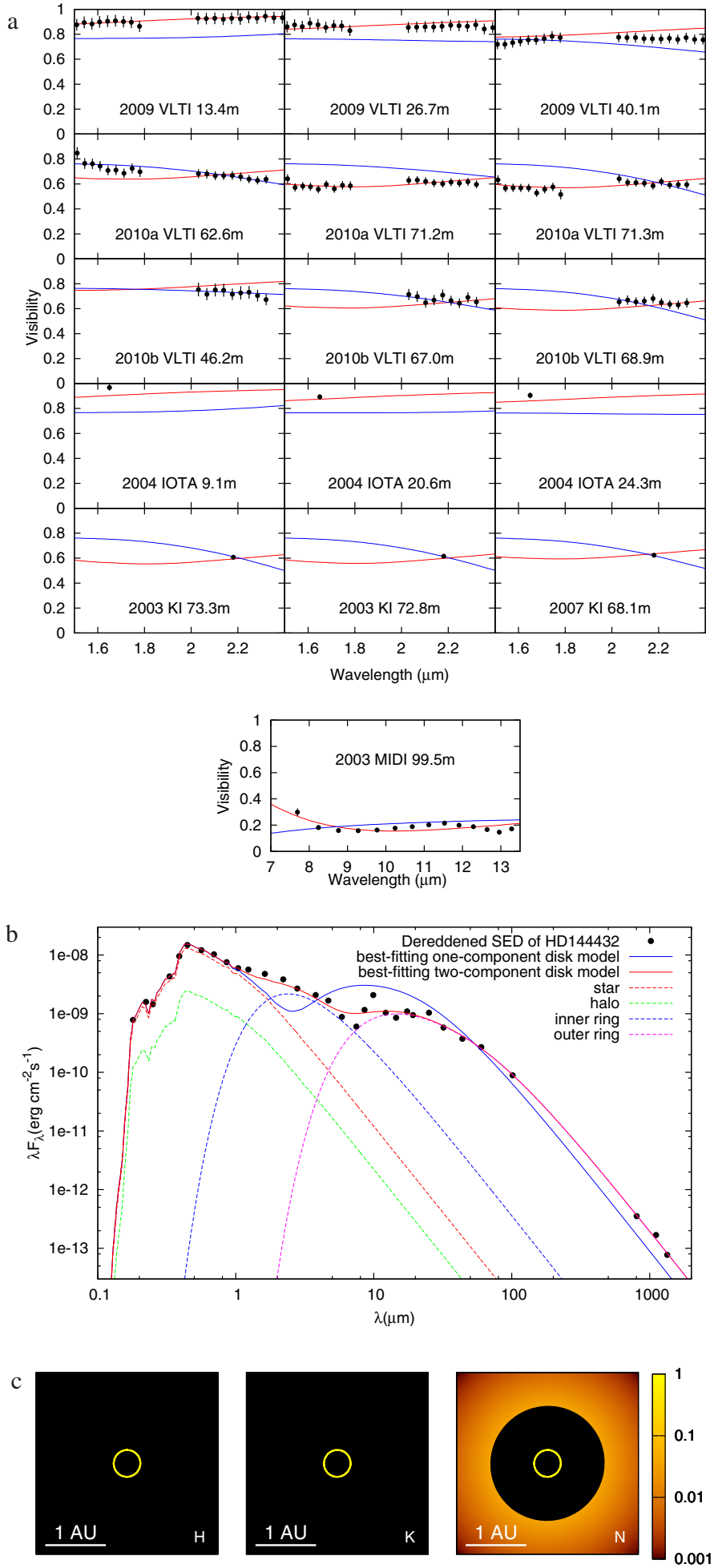

Fig. 3. Comparison of the best-fit two-component disk model (red lines; see Table 3) and best-fit one-component disk model (blue lines) with the observations of HD 144432. a) Near- and mid-infrared visibilities. The data sets 2004 IOTA, 2003 KI, 2007 KI, and 2003 MIDI mentioned in the panels are taken from Monnier et al. (2006), Monnier et al. (2005), Eisner et al. (2009), and Leinert et al. (2004), respectively. b) SED (from Leinert et al. 2004) and temperature-gradient models. The dashed lines denote the contributions from individual components in the best-fit two-component disk model. c) $\mathrm{H}$-, $\mathrm{K}$-, and $\mathrm{N}$-band intensity distributions of the best-fit two-component disk model (Table 3). The second component of the disk, i.e., the more extended part, is visible only in the $N$ band. The star and halo are not plotted. radiation at its local temperature. First, we fitted the SED in the UV/optical band (0.1-1 $\mu \mathrm{m}$ ) with a Kurucz model (Kurucz 1992) to determine the stellar parameters that we should use in our subsequent modeling, i.e., temperature $T_{*}=7180 \mathrm{~K}$, surface gravity $\log g=2.78$, and metallicity $\log m=-0.415$. The seven free parameters in the model are the inclination angle $i$, the position angle $\theta_{\mathrm{D}}$ of the disk's major axis, $T_{\mathrm{in}}, q, r_{\mathrm{in}}$, the radial thickness $\Delta r=r_{\text {out }}-r_{\text {in }}$ of the ring, and the flux fraction $k_{\text {halo }}$.

We tried to find the best-fit model by scanning the physically reasonable parameter range (calculation of approximately $6 \times$ $10^{7}$ models; details of the searching process is described in the Appendix A). Unfortunately, we did not find a model that can reproduce the data reasonably well. The best-fit model (see blue model lines in Fig. 3) deviates significantly from the data, and its $\chi_{\text {red }}^{2}$ is 10.7. This deviation is indicative of a more sophisticated structure of the disk.

\subsection{Temperature-gradient modeling with a two-component disk}

Since the $\chi_{\text {red }}^{2}$ of the one-component disk model discussed above is very large, we introduced a slightly more complicated model in a second modeling step, in which the disk consists of two power-law components, with temperature $T=T_{1, \text { in }}\left(r / r_{1, \text { in }}\right)^{-q_{1}}$ for $r_{1, \text { in }} \leq r \leq r_{1, \text { out }}$ and $T=T_{2 \text {,in }}\left(r / r_{2, \text { in }}\right)^{-q_{2}}$ for $r_{2, \text { in }} \leq$ $r \leq r_{2, \text { out }}$, respectively. We used the subscript 1 to denote the inner disk, and 2 for the outer disk. The assumptions for the star and halo remained unchanged. After computation of several $10^{10}$ models, we found the best-fit solution shown in Table 3 and Fig. 3 (with $\chi^{2}=1.15$; see Appendix A for the searching process).

This best-fit model consists of the star, a halo with a brightness of $18 \%$ of the star, and a nearly face-on $\left(i<28^{\circ}\right)$ twocomponent disk. The inner part of the disk is a thin ring at $r_{1, \text { in }} \sim 0.21 \mathrm{AU}$, with a temperature $T_{1, \text { in }} \sim 1600 \mathrm{~K}$, and a radial thickness $\Delta r_{1} \sim 0.02 \mathrm{AU}$. The power-law index $q_{1}$ was fixed to 0.5 (corresponding to a flared irradiated disk; Kenyon \& Hartmann 1987), since $q_{1}$ cannot be constrained because of the small radial thickness of the inner disk ring (see Appendix A.3). The outer part extends from $\sim 1$ AU to $\sim 10 \mathrm{AU}$, with an inner temperature $\sim 400 \mathrm{~K}$. The contribution from the components to the total NIR flux (at $2 \mu \mathrm{m}$ ) are $60 \%$ from the inner disk, $33 \%$ from the central star, and $6 \%$ from the halo, and almost zero from the outer disk. The contribution from the components to the total MIR flux (at $10 \mu \mathrm{m}$ ) are $21 \%$ from the inner disk, $78 \%$ from the outer disk, and only $1 \%$ from the star and halo. In Fig. 3, we show the comparison of this best-fit model $\left(\chi_{\text {red }}^{2}=1.15\right)$ to our AMBER visibilities together with visibilites from IOTA, Keck, and MIDI measurements, as well as SED data from literature.

A prominent feature of the model is the large gap between the inner and outer disk. The size of the NIR emitting region is confined to $\sim 0.21 \pm 0.01$ AU by the NIR visibilities. The low visibilities in the MIR suggest a much larger size of $\gtrsim 1 \mathrm{AU}$ for the MIR emitting region. If a smooth temperature profile (and hence a continuous emission distribution) is assumed between the two distinct length scales, the NIR emitting region will be much broader than in our model, and would lead to a NIR flux much higher than the observation. Therefore, the gap in our model is strongly required when intepreting all data simultaneously.

\section{Discussion}

To compare the derived NIR sizes with the expected dust sublimation radius and other HAEBE stars, we plot HD 144432 in 
the size-luminosity diagram introduced by Monnier \& MillanGabet (2002) using our $K$-band ring-fit radii (inclination $i=0^{\circ}$ models, see Sect. 3.1 and Table 1) together with the luminosity $L=14.5 \pm 4 L_{\odot}(\mathrm{M} 05)$. Figure 4 shows that the inner ring radius of $0.17 \pm 0.01 \mathrm{AU}$ (star-disk-halo model; adopting a distance of $\sim 145 \pm 20 \mathrm{pc}$, see below) is roughly consistent with the predicted dust-sublimation radius of $0.13 \mathrm{AU}$ corresponding to a sublimation temperature of $1500 \mathrm{~K}$ and gray dust opacities. Furthermore, we compared the measured radius with the prediction of a model including backwarming and accretion luminosity (Millan-Gabet et al. 2007). This model suggests an inner disk radius of $\sim 0.27 \mathrm{AU}$ for a stellar luminosity of $14.5 L_{\odot}$ and an accretion luminosity of $1.0 L_{\odot}$ (Garcia Lopez et al. 2006; we scaled down the value according to the difference in assumed star parameters). Our measured inner-ring radius of $\sim 0.17 \mathrm{AU}$ lies in the range between these two model predictions of $0.13 \mathrm{AU}$ and $0.27 \mathrm{AU}$.

The error bars of the radii for HD 144432 in Fig. 4 represents only the uncertainties in the visibility measurements and do not include the uncertainty in the distance. Pérez et al. (2004) concluded that HD 144432 is likely a member of the star association Sco OB 2-2, with a distance of $\sim 145$ pc. However, in previous studies, distances in the range from $108 \mathrm{pc}$ to $2.4 \mathrm{kpc}$ were also reported (e.g., Pottasch \& Parthasarathy 1988; Pérez et al. 2004). In this paper, we adopted the distance of $145 \pm 20 \mathrm{pc}$ (the error bar corresponds to the distance dispersion within Sco OB 2-2; see Preibisch et al. 2002). In spite of the distance uncertainty, it is possible to discuss the location of HD 144432 relative to the $1500 \mathrm{~K}$ line for the following reason. If the adopted distance is wrong by a certain factor, then this factor changes both the ring-fit radius and the luminosity in such a way that the location of the star moves parallel to the sublimation radius lines in the size-luminosity relation (Monnier \& Millan-Gabet 2002).

Both our geometric and temperature-gradient modeling suggest the existence of a halo component, which contributes $\sim 6-12 \%$ to the total NIR flux in the best-fit geometric model (in the best-fit temperature-gradient model $\sim 6 \%$ at $2 \mu \mathrm{m}$ ). The size of the halo component cannot be measured precisely, but the visibilities suggest that it is $\gtrsim 1$ AU. Given its large distance to the central star, the halo emission is probably dominated by scattered stellar light (Akeson et al. 2005; M06). Plausible origins of the halo material include an infalling remnant envelope, dust entrained in the stellar wind/outflow (M06), or the flaring outer disk, which scatters the stellar light (Pinte et al. 2008).

Our temperature-gradient modeling suggests that the disk consists of two components with distinct length scales. The inner component is a thin ring at an inner radius of $\sim 0.21 \mathrm{AU}$ with a temperature of $\sim 1600 \mathrm{~K}$ and a radial thickness $\sim 0.02 \mathrm{AU}$. The outer part extends from $\sim 1$ AU to $\sim 10$ AU with an inner temperature of $\sim 400 \mathrm{~K}$. The disk is seen roughly face-on with an inclination angle of $i<28^{\circ}$.

The small radial thickness of the inner ring-shaped disk is consistent with the puffed-up rim model (Natta et al. 2001; Dullemond et al. 2001; Dullemond 2002; Dominik et al. 2003; Dullemond \& Dominik 2004). In such a model, the NIR emission is dominated by the puffed-up inner rim at the dust sublimation radius, and the region of the disk behind the rim is colder owing to shadowing effects, which can lead to a gap in the observed intensity distribution. A gap phenomenon was also reported for other Herbig stars (e.g., Benisty et al. 2010; Tatulli et al. 2011). While the NIR emission of the disk is dominated exclusively by the inner ring (presumably the puffed-up rim), the MIR emission has a bimodal distribution, with $\sim 20 \%$ of the emission originating from the inner ring, and the rest from the

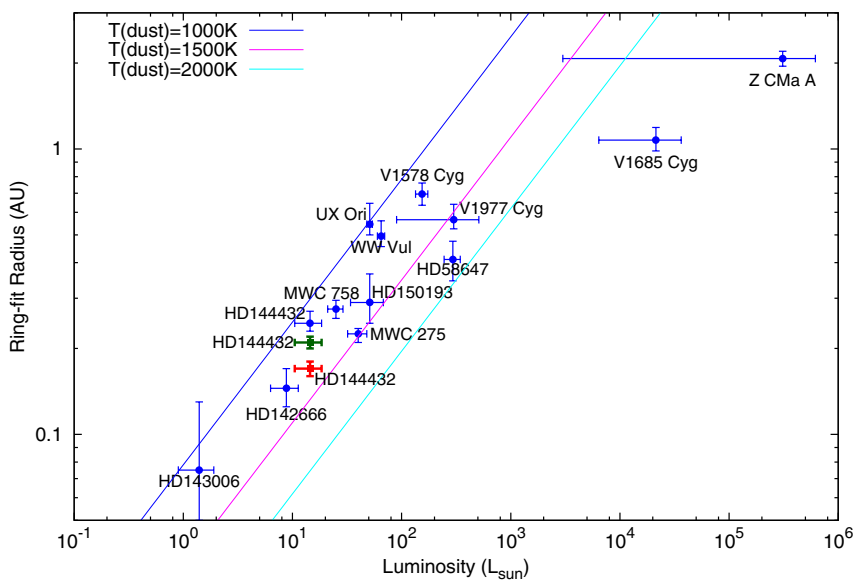

Fig. 4. Size-luminosity diagram for HAeBe stars (Monnier \& MillanGabet 2002). Blue dots: data taken from Monnier et al. (2005). Red square: HD 144432 (ring-fit $K$-band radius of the star-ring-halo uninclined model). Dark-green square: HD 144432 (ring-fit $K$-band radius of the star-ring uninclined model). The error bars of the radii for HD 144432 represent only the uncertainties in the visibilty measurements, and do not include the distance uncertainty. Lines: theoretical dust sublimation radius for gray dust and three different dust sublimation temperatures. Predictions of models including backwarming are discussed in Sect. 4.

outer parts. This spatial distribution of MIR emission is also consistent with the prediction of the rim model (van Boekel et al. 2005).

\section{Summary and conclusions}

We have presented VLTI/AMBER observations of the Herbig Ae star HD 144432 in the $H$ and $K$ bands. The following results were obtained.

The $K$ - and $H$-band emitting regions have geometric ringfit radii of $0.21 \pm 0.01 \mathrm{AU}$ and $0.20 \pm 0.01 \mathrm{AU}$, respectively. When we introduced an additional halo component, we obtained the smaller disk ring-fit radii of $0.17 \pm 0.01 \mathrm{AU}$ ( $K$ band) and $0.18 \pm 0.01 \mathrm{AU}$ ( $H$ band). This measured $K$-band ring radius of $\sim 0.17 \mathrm{AU}$ lies in the range between the aforementioned dust sublimation radius of $\sim 0.13 \mathrm{AU}$ (size-luminosity relation in Fig.4; for sublimation temperature of $1500 \mathrm{~K}$ and gray dust), and the prediction of models including backwarming ( 0.27 AU). Both our geometric and temperature-gradient modeling indicate the existence of an additional extended halo component. In the best-fit temperature-gradient model, the halo contributes $\sim 6 \%$ at $2 \mu \mathrm{m}$.

Our best-fit temperature-gradient disk model can approximately reproduce both the NIR-MIR visibilities and the SED data (Fig. 3). The model consists of the central star, an extended halo, and a nearly face-on two-component disk. In the modeling procedure, we started with a very wide range for all model parameters, calculated several $10^{10}$ models corresponding to all combinations of the parameters, and finally obtained disk parameters that seem to be physically quite reasonable. The inner part of the disk is a thin ring at an inner radius of $\sim 0.21 \mathrm{AU}$ with a temperature of $\sim 1600 \mathrm{~K}$ and a radial thickness $\sim 0.02 \mathrm{AU}$. The outer part extends from $\sim 1$ AU to $\sim 10$ AU with an inner temperature of $\sim 400 \mathrm{~K}$. The NIR emission of the disk is dominated by the inner ring. The MIR emission has a bimodal distribution, with $\sim 20 \%$ of the emission originating from the inner ring and 
Table A.1. Scanned parameter space in the two-component disk temperature-gradient modeling. Upper part: one-component disk model. Lower part: two-component disk model.

\begin{tabular}{ccccccccccc}
\hline \hline Step & $i\left(^{\circ}\right)$ & $\theta_{\mathrm{D}}\left({ }^{\circ}\right)$ & $T_{1, \text { in }}(\mathrm{K})$ & $q_{1}$ & $r_{1, \text { in }}(\mathrm{AU})$ & $\Delta r_{1}(\mathrm{AU})$ & $T_{2, \text { in }}(\mathrm{K})$ & $q_{2}$ & $r_{2, \text { in }}(\mathrm{AU})$ & $\Delta r_{2}(\mathrm{AU})$ \\
\hline 1 & $0-60$ & $-90-90$ & $800-3000$ & $0-3$ & $0.05-0.5$ & $0.001-5$ & & & \\
\hline 1 & $0-60$ & $-90-90$ & $800-3000$ & $0-3$ & $0.05-0.5$ & $0.001-5$ & $100-700$ & $0-3$ & $0.2-10$ & $0.05-50$ \\
2 & $0-50$ & $-90-90$ & $1000-2500$ & $0-3$ & $0.15-0.4$ & $0.003-0.1$ & $250-500$ & $0.2-1.8$ & $0.4-3$ & $2.0-50$ \\
3 & $0-40$ & $-90-90$ & $1200-2000$ & $0-3$ & $0.15-0.3$ & $0.008-0.04$ & $350-450$ & $0.5-1.3$ & $0.6-1.2$ & $4.0-15$ \\
4 & $0-35$ & $-90-90$ & $1300-1900$ & $0-3$ & $0.18-0.3$ & $0.010-0.03$ & $360-440$ & $0.6-1.1$ & $0.7-1.2$ & $5.0-12$ \\
5 & $0-30$ & $-90-90$ & $1400-1800$ & 0.5 & $0.18-0.25$ & $0.014-0.024$ & $360-420$ & $0.7-1.0$ & $0.8-1.1$ & $6.0-10$ \\
6 & $0-30$ & $-90-90$ & $1500-1700$ & 0.5 & $0.2-0.23$ & $0.016-0.022$ & $370-410$ & $0.75-0.9$ & $0.85-1.0$ & $7.0-9$ \\
\hline
\end{tabular}

the rest from the outer parts. The temperature-gradient modeling suggests an upper limit for the inclination angle of $i<28^{\circ}$.

Acknowledgements. We thank the ESO VLTI team on Paranal for the excellent collaboration. The data presented here were reduced using the publicly available data-reduction software package amdlib kindly provided by the JeanMarie Mariotti Center (http://www.jmmc.fr/datar_processing_amber. $\mathrm{htm}$ ). This publication makes use of the SIMBAD database operated at CDS, Strasbourg, France. Finally, we thank the anonymous referee for his helpful comments.

\section{Appendix A: Searching for the best-fit models}

\section{A.1. General description of the searching method}

We searched for the best-fit one-component and two-component models describing the visibility and SED data. We established a grid in the multi-dimensional parameter space, and evaluated the $\chi^{2}$ on each grid point to find the $\chi^{2}$-minimum. A large parameter range was first scanned to roughly locate the global minimum. In further processing, we then computed narrower grids around the global minimum to confine the parameters with higher precision. In each step, we computed the models for all combinations of all parameters.

We treated the parameter $k_{\text {halo }}$ in the following way. For each combination of all the other parameters, the modeled visibilities depend linearly on the halo fraction $k_{\text {halo }} /\left(1+k_{\text {halo }}\right)$, while the modeled fluxes are independent of $k_{\text {halo }}$. Therefore, the best-fit value of $k_{\text {halo }}$ can be found with linear regression.

\section{A.2. Searching for the best-fit one-component disk model}

We searched for the best-fit one-component disk model within the wide parameter ranges listed in Table A.1. We divided the range of each parameter into 20 grid points and computed $20^{6} \approx$ $6 \times 10^{7}$ models for all combinations of all parameters. No model with reasonable fitting could be found.

\section{A.3. Searching for the best-fit two-component disk model}

When searching for the best-fit two-component disk model, we start from wide parameter ranges and gradually zoom in (see the ranges listed in Table A.1). The best-fit parameters are listed in Table 3. Owing to the small radial thickness of the inner ring, fits of equal quality ( $\operatorname{similar} \chi^{2}$ values) can be found for each $q_{1}$ value (see the $\chi^{2}$ map in Fig. A.1). Therefore, we set $q_{1}=0.5$ (corresponding to a flared irradiated disk; Kenyon \& Hartmann 1987) in the last two search steps.
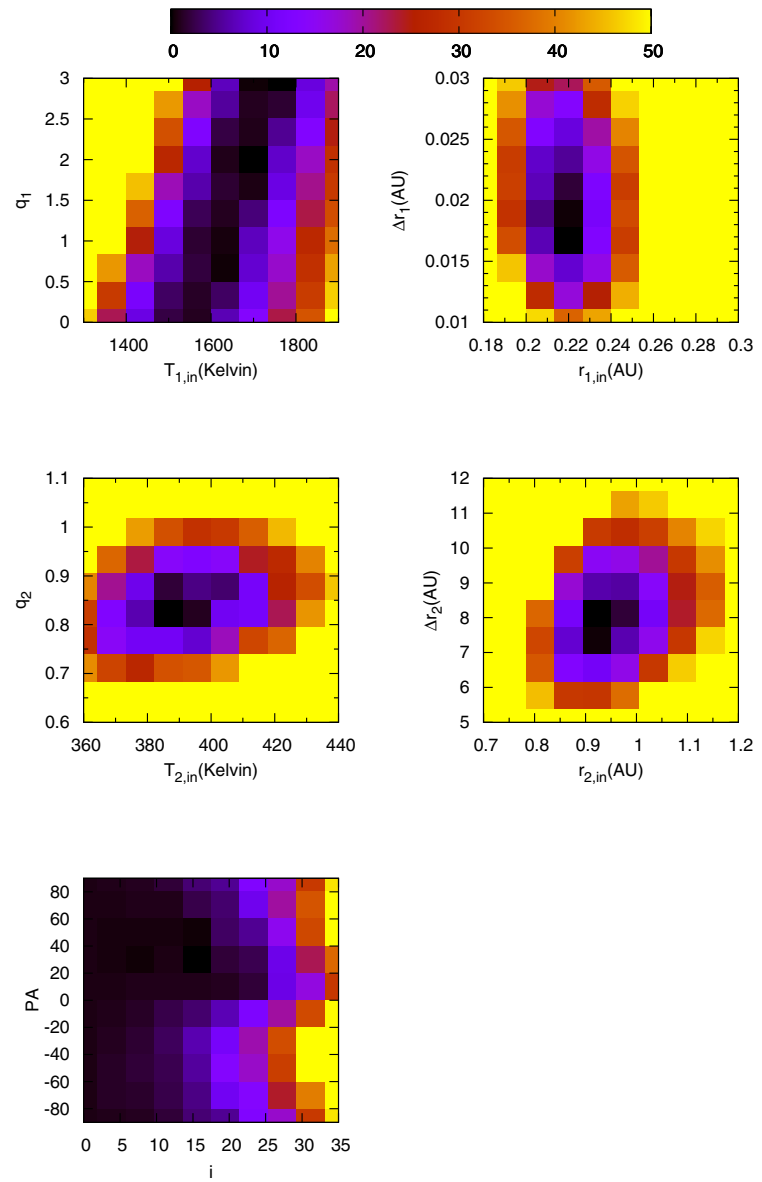

Fig. A.1. $\chi^{2}$ maps showing $\Delta \chi^{2}=\chi^{2}-\chi_{\min }^{2}$ as function of the parameters in the two-componet disk model (results of the searching step 4). For each subset of parameters, the $\Delta \chi^{2}$ shown is the lowest value of all combinations of other parameters.

In each scanning step, we divided the range of each parameter into 10 grid points (except $q_{1}$ in the last two steps) and computed the models for all combinations of all parameters. In total, we computed $4.2 \times 10^{10}$ models.

\section{References}

Acke, B., \& van den Ancker, M. E. 2004, A\&A, 426, 151

Akeson, R. L., Walker, C. H., Wood, K., et al. 2005, ApJ, 622, 440

Benisty, M., Tatulli, E., Ménard, F., \& Swain, M. R. 2010, A\&A, 511, A75 Carmona, A., van den Ancker, M. E., \& Henning, T. 2007, A\&A, 464, 687 
Dominik, C., Dullemond, C. P., Waters, L. B. F. M., \& Walch, S. 2003, A\&A, 398,607

Dullemond, C. P. 2002, A\&A, 395, 853

Dullemond, C. P., \& Dominik, C. 2004, A\&A, 417, 159

Dullemond, C. P., Dominik, C., \& Natta, A. 2001, ApJ, 560, 957

Eisner, J. A., Lane, B. F., Hillenbrand, L. A., Akeson, R. L., \& Sargent, A. I. 2004, ApJ, 613, 1049

Eisner, J. A., Graham, J. R., Akeson, R. L., \& Najita, J. 2009, ApJ, 692, 309

Garcia Lopez, R., Natta, A., Testi, L., \& Habart, E. 2006, A\&A, 459, 837

Hillenbrand, L. A., Strom, S. E., Vrba, F. J., \& Keene, J. 1992, ApJ, 397, 613

Keller, L. D., Sloan, G. C., Forrest, W. J., et al. 2008, ApJ, 684, 411

Kenyon, S. J., \& Hartmann, L. 1987, ApJ, 323, 714

Kreplin, A., Kraus, S., Hofmann, K.-H., et al. 2012, A\&A, 537, A103

Kurucz, R. L. 1992, in The Stellar Populations of Galaxies, ed. B. Barbuy, \& A. Renzini, IAU Symp., 149, 225

Leinert, C., van Boekel, R., Waters, L. B. F. M., et al. 2004, A\&A, 423, 537

Malfait, K., Bogaert, E., \& Waelkens, C. 1998, A\&A, 331, 211

Meeus, G., Waelkens, C., \& Malfait, K. 1998, A\&A, 329, 131

Meeus, G., Waters, L. B. F. M., Bouwman, J., et al. 2001, A\&A, 365, 476

Millan-Gabet, R., Malbet, F., Akeson, R., et al. 2007, Protostars and Planets V, 539
Monnier, J. D., \& Millan-Gabet, R. 2002, ApJ, 579, 694

Monnier, J. D., Millan-Gabet, R., Billmeier, R., et al. 2005, ApJ, 624, 832

Monnier, J. D., Berger, J., Millan-Gabet, R., et al. 2006, ApJ, 647, 444

Monnier, J. D., Tuthill, P. G., Ireland, M., et al. 2009, ApJ, 700, 491

Natta, A., Prusti, T., Neri, R., et al. 2001, A\&A, 371, 186

Pasinetti Fracassini, L. E., Pastori, L., Covino, S., \& Pozzi, A. 2001, A\&A, 367 521

Pérez, M. R., van den Ancker, M. E., de Winter, D., \& Bopp, B. W. 2004, A\&A, 416,647

Petrov, R. G., Malbet, F., Weigelt, G., et al. 2007, A\&A, 464, 1

Pinte, C., Ménard, F., Berger, J. P., Benisty, M., \& Malbet, F. 2008, ApJ, 673, L63

Pottasch, S. R., \& Parthasarathy, M. 1988, A\&A, 192, 182

Preibisch, T., Brown, A. G. A., Bridges, T., Guenther, E., \& Zinnecker, H. 2002, AJ, 124, 404

Sylvester, R. J., Skinner, C. J., Barlow, M. J., \& Mannings, V. 1996, MNRAS, 279,915

Tatulli, E., Millour, F., Chelli, A., et al. 2007, A\&A, 464, 29

Tatulli, E., Benisty, M., Ménard, F., et al. 2011, A\&A, 531, A1

Thé, P. S., de Winter, D., \& Perez, M. R. 1994, A\&AS, 104, 315

van Boekel, R., Dullemond, C. P., \& Dominik, C. 2005, A\&A, 441, 563 